\title{
Cost-Aware Fronthaul Rate Allocation to Maximize Benefit of Multi-User Reception in C-RAN
}

\author{
Dora Boviz $^{* \dagger}$, Chung Shue Chen*, Sheng Yang ${ }^{\dagger}$ \\ *Nokia Bell Labs \\ 7 Route de Villejust, 91620 Nozay, France \\ †CentraleSupélec, Laboratoire des Signaux et Systèmes \\ 3 rue Joliot-Curie, 91190 Gif-sur-Yvette, France \\ E-mails: dora.boviz@nokia-bell-labs.com, \\ chung_shue.chen@nokia-bell-labs.com, sheng.yang@centralesupelec.fr
}

\begin{abstract}
Aiming to throughput enhancement in future mobile networks, dense deployment of wireless access points (APs) is intended. Spectral efficiency on the uplink can be improved via joint processing of users located in areas covered by several APs. Thanks to centralized processing of such users enabled by Cloud Radio Access Network (C-RAN) architecture, low-latency multi-cell cooperation becomes possible. However, the price to pay to benefit from its advantages is the cost of transferring data from distributed access points to the data center through limited-capacity fronthaul links. A mobile network operator using C-RAN would have the objective to maximize data transmission rates with lowest possible fronthaul rate. We consider in this work the optimal tradeoff between the amount of fronthaul allocated and the sum rate of each user group in a multiple access scheme, where the wireless resource is reused among the user groups. By performing low-complexity joint reception of signals quantized according to attributed fronthaul rate, we can maximize the benefit of uplink transmission in C-RAN despite constrained fronthaul links. With optimal fronthaul allocation, the net benefit of the uplink transmission improves by $10 \%$ in a usual configuration.
\end{abstract}

\section{INTRODUCTION}

The idea of offloading base-band functions from several cell sites to a centralized data center arises the great opportunity of joint signal processing, but also the practical challenge of data transfer between remote Access Points (APs) and the Central Office (CO). Furthermore, in $5 \mathrm{G}$ architecture, centralization is identified as a key disruption [1], since it enables user-centric processing which can greatly facilitate multi-cell cooperation. Finding an efficient and affordable data transfer solution over limited capacity fronthaul (FH) links between APs and the CO is crucial to realize Cloud Radio Access Network (C-RAN) deployments and fulfill $5 \mathrm{G}$ requirements [2].

Mobile network operators have to offer better Qualityof-Service (QoS) at lower cost, thus the efficient use of wireless spectrum, APs, and computational resources become more important then ever. In this paper, we will propose an optimized transmission scheme for C-RAN to increase uplink (UL) transmission efficiency from the User Equipments (UEs) to the end of physical layer (PHY) processing. To enhance spectral efficiency, low latency multi-cell cooperation is realized through partially non-orthogonal uplink scheduling. We aim to benefit from Multiple Input Multiple Output (MIMO) enabled APs and centralization of signal processing while keeping user coordination and receiver complexity low. To deal with limited capacity fronthaul links between the APs and the $\mathrm{CO}$, it is important to optimize the fronthaul rate allocation in order to adapt quantization level of transferred signals according to the quality of the wireless transmission.

Constrained Non-Orthogonal Multiple-Access (NOMA) is proposed in [3] for uplinks where users are organized into several subsets and within each of them, the users transmit simultaneously over the same wireless channel, whereas orthogonal resources are allocated to each group in order to eliminate the interference between the groups. On the receiver side, one can use Minimum Mean Square Error (MMSE) detection to separate signals of users transmitting together. Thanks to the limited number of users in each group, the receiver complexity can be relatively low compared to that needed with fully nonorthogonal transmission of all users. Maximizing the sum rate of UL multi-user transmission through enhanced user association is described in [4], where the authors consider channel estimation error and compare different methods for sum rate improvement. Joint fronthaul and power allocation in fully orthogonal multi-user model is studied in [5]. The authors provide practical quantization scheme and show that the optimization of fronthaul allocation improves significantly the overall throughput compared to uniform distribution of available fronthaul. The improvement is obtained by the novel scheme which takes into account different link gains of received signals that are forwarded on the same fronthaul link. Impact of signal compression in C-RAN architecture to satisfy fronthaul constraint is widely studied, see for example [6]. In our previous work [7], we have characterized the optimal fronthaul rate for a single MIMO channel. In this paper, we extend the optimization to a system of several user groups that transmit independently and share the fronhaul links. We show that optimally distributing available fronthaul rate between multi-user transmissions allows to improve the overall efficiency of the transmission. In an typical scenario, end-to-end benefit increases by $10 \%$ thanks to the proposed tradeoff between sum rate and fronthaul usage.

The rest of the paper is organized as follows. We describe the system model and the multi-user transmission scheme in 


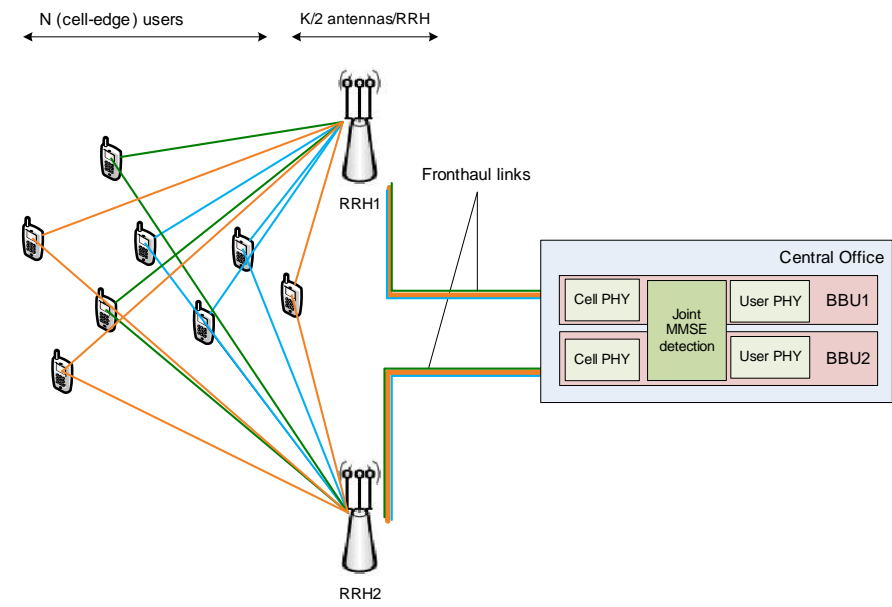

Fig. 1: System model with several user groups (example $M=$ 2 RRHs), where JD stands for joint detection and BBU stands for base-band unit.

Section II. Then, in Section III we develop the metric linking system performance to the fronthaul rate. The optimization problem of fronthaul allocation is formulated in Section IV. Section V provides numerical evaluation of the proposed fronthaul allocation scheme and observations. Finally, Section VI contains the conclusion.

\section{System Model}

The system that we study is depicted in Figure 1. We consider a C-RAN system with $M$ RRHs located at the cellsites with $a$ antennas each, thus the total number of antennas is $K=a \cdot M$. All of the RRHs are connected to the same $\mathrm{CO}$ where the major part of signal processing is realized for the cells. We assume that all the $N$ users are located in the cell-edge region and are able to communicate with all the $M$ RRHs. To avoid interference between them, they are benefiting from joint detection (JD) at the receiver. In the system that we study, user-PHY functions of all the $N$ users are placed in the $\mathrm{CO}$ in order to enable multi-cell joint processing on the uplink [8].

The users can be associated to each other randomly or in a deterministic way to create the user groups to which we allocate a wireless resource. Orthogonal resource blocks (RBs) are allocated to different groups, but users who transmit using the same RB will interfere with each other. The RRHs, after receiving the combination of uplink signals transmitted by every group, will forward the quantized version of this signal to the $\mathrm{CO}$ through limited-capacity fronthaul links. The total capacity of each fronthaul link is shared among all of the user groups.

We assume a block fading channel with a coherence time that is long enough to consider allocating fronthaul capacity to the groups using real-time channel realizations. In terms of user mobility, e.g., for pedestrian users moving at $5 \mathrm{~km} / \mathrm{h}$, the transmission characteristics change approximately every 720 ms, which is a time window large enough to update network parameters.

We use the following notational conventions: for random variables, upper case letters with bold and non-italic fonts, e.g., $\mathbf{V}$, for vectors, and bold and sans serif fonts, e.g., $\mathbf{M}$, for matrices. Deterministic quantities are denoted with italic letters, e.g., a scalar $x$, lowercase bold for a vector $\boldsymbol{v}$, and uppercase bold letters for a matrix $\boldsymbol{M}$. Logarithms are in base 2 and superscript $(.)^{H}$ denotes the conjugate transpose of a vector or a matrix.

We have $N$ users uniformly distributed in the region covered by every RRH. The channel of each user $n \in\{1, \ldots, N\}$ towards all antennas at both RRHs is denoted by $\boldsymbol{h}_{n}$ which is a $K$ dimensional array following the Gaussian distribution $\mathcal{N}\left(0, \boldsymbol{R}_{n}\right)$ with $\boldsymbol{R}_{n} \in \mathbb{C}^{K \times K}$.

The number of user groups is denoted $L$, there are $s_{l}$ users in the group $l \in\{1, \ldots, L\}$. The multi-user channel of a group towards the whole set of antennas is the $K \times s_{l}$ dimensional matrix $\mathbf{H}_{l}$. We assume that the channel is perfectly known at the receiver. Though, channel estimators that are generally used result in an estimation error, its impact on the fronthaul allocation is not significant.

The Gaussian channel noise vector is denoted $\boldsymbol{n}_{l} \sim$ $\mathcal{N}\left(0, \sigma_{z}^{2} \boldsymbol{I}_{K}\right)$. Power of the input signal is normalized, so that noise covariance scales with $\sigma_{z}^{2}=\frac{1}{\mathrm{SNR}}$.

The received signal for group $l$ by the whole set of antennas is the $K$-dimensional vector $\boldsymbol{y}_{l}$ and it is given by the superposition of the signals sent by all of the $s_{l}$ users in the group denoted by $\Pi_{l}=\left\{\pi_{1}^{l}, \ldots, \pi_{s_{l}}^{l}\right\}$.

$$
\boldsymbol{y}_{l}=\sum_{i=1}^{s_{l}} \boldsymbol{h}_{\pi_{i}^{l}} x_{\pi_{i}^{l}}+\boldsymbol{n}_{l}
$$

The fronthaul capacity of the link between the $m$-th RRH and the $\mathrm{CO}$ allocated to group $l$ is denoted by $c_{m}^{(l)}$ with $l \in\{1, \ldots, L\}$ and $m \in\{1, \ldots, M\}$. We can write this total fronthaul rate available using fronthaul rates $c_{l k}$ dedicated to the transmission of the signal from group $l$ received by antenna $k$ located at RRH $m$ as

$$
c_{m}^{(l)}=\sum_{k=(m-1) \cdot a+1}^{m \cdot a} c_{l k} .
$$

We denote by $\boldsymbol{c}^{(l)}=\left\{c_{l 1}, \ldots c_{l K}\right\}$ the set of capacity values attributed to group $l$ and $\bar{c}_{m}$ the total fronthaul capacity available between RRH $m$ and the CO.

\section{SUM RATE OF MULTI-USER TRANSMisSION With LIMITED FRONTHAUL}

Once user groups are created and scheduled, users transmit following the scheduling decisions and RRHs receive the signals of every group - being the superposition of the signals transmitted by all the UEs in the group - and forward frequency-domain I/Q symbols to the $\mathrm{CO}$. We perform receive Fast Fourier Transform in the RRH to decorrelate the subcarriers and enable to select the ones that need to be forwarded 
(since they are required for the JD). This decorrelation improves also the throughput by reducing quantization noise in the forwarded signal.

The channel gain depends on subcarrier frequency, so the fronthaul allocation can change with a different scheduling decision or a modification of user groups. In the following computations, we consider only one subcarrier per group, but the extension to several subcarriers is straightforward.

\section{A. Quantization noise}

We define in the following the equivalent quantization noise with limited capacity fronthaul for a group with $s_{l}$ users transmitting towards $K$ receive antennas. The achievable rate with a fronthaul capacity $c_{l k}$ can be defined using the mutual information between the received signal and the forwarded one, given the distortion between them.

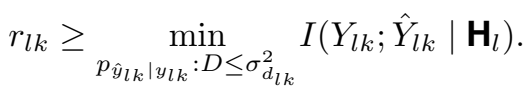

We can derive the following relation between the received signal power and the variance of the distortion noise

$$
\sigma_{d_{l k}}^{2} \leq \sigma_{y_{l k} \mid \mathbf{H}_{l}}^{2} 2^{-c_{l k}}
$$

where

$$
\sigma_{y_{l k} \mid \mathbf{H}_{l}}^{2}=\sum_{i=1}^{s_{l}}\left(\left|h_{k \pi_{i}^{l}}\right|^{2}\right)+\sigma_{z}^{2} .
$$

We use a scaling factor $\alpha_{l k}$ in order to adapt the power of forwarded signal to the fronthaul capacity used, i.e.,

$$
\alpha_{l k}=\frac{\sigma_{y_{l k} \mid \mathbf{H}_{l}}^{2}-\sigma_{d_{l k}}^{2}}{\sigma_{y_{l k} \mid \mathbf{H}_{l}}^{2}} \forall k \in\{1, \ldots, K\} .
$$

Scaling factors for each antenna form the matrix $A_{l}=$

diag $\left(\alpha_{l k}\right)$. The distortion is then characterized by the $k=\{1, \ldots, K\}$

following upper bound:

$$
\begin{aligned}
\frac{\sigma_{d_{l k}}^{2}}{\alpha_{l k}}= & \frac{\sigma_{d_{l k}}^{2} \sigma_{y_{l k} \mid \mathbf{H}_{l}}^{2}}{\sigma_{y_{l k} \mid \mathbf{H}_{l}}^{2}-\sigma_{d_{l k}}^{2}} \leq \frac{\sigma_{y_{l k} \mid \mathbf{H}_{l}}^{2} 2^{-c_{l k}}}{1-2^{-c_{l k}}}, \\
& \forall k \in\{1, \ldots, K\}, \forall l \in\{1, \ldots, L\} .
\end{aligned}
$$

\section{B. Achievable sum rate}

We can compute the achievable sum rate for a given joint transmission group using the mutual information between the signal sent by all users in the group and the one received in the $\mathrm{CO}$ :

$$
\sum_{i=1}^{s_{l}} r_{i} \geq I\left(\mathbf{X}_{l} ; \hat{\mathbf{Y}}_{l} \mid \mathbf{H}_{l}\right)=h\left(\mathbf{X}_{l}\right)-h\left(\mathbf{X}_{l} \mid \hat{\mathbf{Y}}_{l}, \mathbf{H}_{l}\right)
$$

We compute both entropy terms in order to find the lower bound of the sum rate. The first term describes the quantity of information sent by the users, and thus depends on the transmission power:

$$
h\left(\mathbf{X}_{l}\right)=\log \left(\operatorname{det}\left(2 \pi e \mathbb{E}\left[\mathbf{X}_{l} \mathbf{X}_{l}^{H}\right]\right)\right)=\log ((2 \pi e))
$$

The second term comes from the loss of information between the UEs and the $\mathrm{CO}$ and can be computed using the linear MMSE covariance $\mathcal{C}_{e}$.

$$
h\left(\mathbf{X}_{l} \mid \hat{\mathbf{Y}}_{l}, \mathbf{H}_{l}\right) \leq \log \left(\operatorname{det}\left(2 \pi e \mathcal{C}_{e}\right)\right) .
$$

For this, we use the definition of received signal by the $\mathrm{CO}$ :

$$
\hat{\boldsymbol{y}}_{l}=\boldsymbol{A}_{l}\left(\sum_{i=1}^{s_{l}} \boldsymbol{h}_{\pi_{i}^{l}} \boldsymbol{x}_{i}+\boldsymbol{n}_{l}\right)
$$

where $n=z+d$ is the equivalent noise containing Gaussian channel noise and the quantization noise. The covariance matrix of this equivalent noise is $\mathcal{C}_{N}=\sigma_{z}^{2} I_{K}+$ $\underset{k=\{1, \ldots, K\}}{\operatorname{diag}}\left(\frac{\sigma_{d l k}^{2}}{\alpha_{l k}}\right)$.

We can compute the linear MMSE covariance based on the channel matrix

$$
\mathcal{C}_{e}=\boldsymbol{I}_{s_{l}}-\boldsymbol{H}_{l}^{H}\left(\boldsymbol{H}_{l} \boldsymbol{H}_{l}^{H}+\mathcal{C}_{N}\right)^{-1} \boldsymbol{H}_{l} .
$$

Using the inversion lemma on the lower bound of the mutual information we get the following expressing of the achievable sum rate with a givel channel matrix $\boldsymbol{H}_{l}$

$$
\sum_{i=1}^{s_{l}} r_{i} \geq \log \operatorname{det}\left(I_{s_{l}}+\boldsymbol{H}_{l}^{H} \mathcal{C}_{N}^{-1} \boldsymbol{H}_{l}\right) .
$$

From (7) and (13), we get the achievable sum rate of a group of $N$ users transmitting towards $K$ receive antennas, each of them using $c_{j}, j \in\{1, . . K\}$ bits of fronthaul.

$$
\sum_{i=1}^{s_{l}} r_{i} \geq \log \operatorname{det}\left(I_{s_{l}}+\boldsymbol{H}_{l}^{H} \boldsymbol{V}_{s_{l}}^{-1} \boldsymbol{H}_{l}\right)
$$

with the equivalent noise covariance:

$$
\boldsymbol{V}_{s_{l}}=\sigma_{z}^{2} \boldsymbol{I}_{K}+\underset{k=\{1, \ldots, K\}}{\operatorname{diag}}\left(\frac{\sigma_{y_{l k} \mid \mathbf{H}_{l}}^{2} 2^{-c_{l k}}}{1-2^{-c_{l k}}}\right) .
$$

\section{Fronthaul Allocation Optimization}

After the $\mathrm{CO}$ receives forwarded signals from the RRHs, we can compute the sum rate (14) of every user group using channel realizations in order to evaluate the performance of the multi-user reception in C-RAN under the constraint of limited fronthaul capacity. Using the sum rate expression (14), our aim is to maximize the benefit of the transmission given a constraint on total rate available at each fronthaul link. We provide here a metric enabling the allocation of fronthaul capacity to user groups, which should allow to maximize the efficiency of the transmission. When the optimal fronthaul allocation for each fronthaul link is computed, quantization of forwarded signal is adapted at the RRHs, in order to improve the performance of the complete transmission. 


\section{A. Objective function: net benefit of the transmission}

We use the upper bound of the sum rate (14) to formulate the objective function allowing to maximize the end-to-end benefit of the uplink transmission of $N$ users forming $L$ groups towards the $M$ RRHs with $a$ antennas each. The parameters of this function are the following:

- The Gaussian channel noise variance $\sigma_{z}^{2}$.

- The average received signal power from group $l$ at antenna $k$ given the channel of the group $l: \sigma_{y_{l} \mid}^{2} \mid \mathbf{H}_{l}$.

- The fronthaul capacity $c_{l k}$ with $k \in\{1, \ldots, K\}$ used to forward to the $\mathrm{CO}$ digital base-band I/Q symbols of group $l$ received by antenna $k$. We denote $\boldsymbol{c}^{(l)}=\left(c_{l 1}, \ldots c_{l K}\right)^{T}$ the set of capacity values for group $l$.

- The cost $\lambda_{k}$ of the fronthaul capacity used for the transmission of the signal received on antenna $k$.

In this work we consider only linear fronthaul cost corresponding to exploitation costs such as transmission energy or cost renting the needed transmission rate from the owner of the fronthaul infrastructure. Note that any non-negative convex cost function can be used following the deployment scenario considered.

The following function characterizes the net benefit of the transmission of group $l$ towards the whole set of receive antennas when the fronthaul capacity allocated to the group is $c_{m}^{(l)}=\sum_{k=(m-1) \cdot a+1}^{m \cdot a} c_{l k}$ for the fronthaul link between RRH $m$ and the CO. Given the parameters $\sigma_{z}^{2}, \sigma_{y_{l k} \mid \mathbf{H}_{l}}^{2}, \lambda_{k} \forall k \in$ $\{1, \ldots, K\}$,

$$
f\left(\boldsymbol{H}_{l}, \boldsymbol{c}^{(l)}\right)=\log \operatorname{det}\left(I_{s_{l}}+\boldsymbol{H}_{l}^{H} \boldsymbol{V}_{s_{l}}^{-1} \boldsymbol{H}_{l}\right)-\sum_{k=1}^{K} \lambda_{k} c_{l k}
$$

where $V_{s_{l}}$ is defined in (15). The first term of the function $f($. in (16) gives the instantaneous sum rate during the coherence time block where the channel matrix $\boldsymbol{H}_{l}$ holds and the second term is the total cost of the fronthaul transmission for group $l$ over the whole set of fronthaul links connecting the RRHs to the $\mathrm{CO}$.

\section{B. Limited fronthaul link rate}

First, we aim to find the optimal capacity allocation $\left\{\boldsymbol{c}^{(1) *}, \ldots, \boldsymbol{c}^{(L) *}\right\}$ that maximizes the overall benefit of the uplink transmission of the $N$ users distributed in $L$ groups while the fronthaul capacity used for all groups between each RRH $m$ and the CO is not more than $\bar{c}_{m}$. We need to solve the optimization problem including this constraint when fronthaul links are physically limited to a given rate, for example in low capacity deployments using other transport solution than optical fibers or when the allocation of rates between various services exploiting the same transport links is fixed.

$$
\begin{aligned}
& \text { Find }\left\{\boldsymbol{c}^{(1) *}, \ldots, \boldsymbol{c}^{(L) *}\right\}=\underset{\left\{\boldsymbol{c}^{(1)}, \ldots, \boldsymbol{c}^{(L)}\right\}}{\operatorname{argmax}} \sum_{l=1}^{L} f\left(\boldsymbol{H}_{l}, \boldsymbol{c}^{(l)}\right) \\
& \text { subject to } \sum_{l=1}^{L} c_{m}^{(l)} \leq \bar{c}_{m} \forall m \in\{1, \ldots, M\} .
\end{aligned}
$$

Let us recall that $c_{m}^{(l)}=\sum_{k=(m-1) \cdot a+1}^{m \cdot a} c_{l k}$, so the above constraint can also be written as

$$
\sum_{l=1}^{L} \sum_{k=(m-1) \cdot a+1}^{m \cdot a} c_{l k} \leq \bar{c}_{m} \forall m \in\{1, \ldots, M\}
$$

Proposition 1. The problem (17) is concave, thus admits a unique solution that gives the optimal capacity allocation scheme.

Proof: The constraint in (17) is linear, as well as the cost term in (16), thus the concavity of the first term of $f($.$) is$ sufficient to show that the problem is concave. Notice that the second term of (16), i.e., $\sum_{k=1}^{K} \lambda_{k} c_{l k}$ is linear w.r.t. $\boldsymbol{c}^{(l)}$, so it can be considered as concave. The function $\log \operatorname{det}(\boldsymbol{A})$ is concave if and only if the matrix $A$ is non-negative definite. The sum of two non-negative definite matrices is also non-negative definite. Since the identity matrix satisfies this condition, we only need to show that the second term of the argument of the $\log \operatorname{det}($.$) in (16) is non-negative definite. The equivalent$ SNR matrix $\boldsymbol{V}_{s_{l}}$ is diagonal with positive elements which are its eigenvalues, thus it is positive definite. This property stands also for its inverse.

If a positive definite matrix $M$ is multiplied by another matrix and its hermitian as $\boldsymbol{B}^{H} \boldsymbol{M} \boldsymbol{B}$, the result is also positive definite if $\boldsymbol{B}$ is full rank. This is true for $\boldsymbol{H}_{l}^{H} \boldsymbol{V}_{s_{l}}^{-1} \boldsymbol{H}_{l}$ since the columns of $\boldsymbol{H}$ are independent, thus $\operatorname{rank}(\boldsymbol{H})=s_{l}$. Consequently, the matrix being the argument of $\log \operatorname{det}($.$) is$ positive definite and also non-negative definite, thus the first term of (16) which implies with the above reasons that (17) is concave.

\section{Unconstrained fronthaul links}

In some deployment scenarios, very high capacity fronthaul links are available between RRHs and the CO. These can be considered in practice as unlimited links. For example, when optical fibers with Dense Wavelength Division Multiplexing are used, they can satisfy bandwidth requirements of mobile fronthaul, but installation and consequently usage cost may be high. Unconstrained optimization of the benefit of uplink transmission would allow to allocate fronthaul optimally for a given fronthaul cost.

Without constraint on the maximal fronthaul rate available at each link, to solve the optimization problem we need to maximize the same concave function as in (17). Intervals of possible fronthaul capacity values are specified according to the transmission scheme, see below (19). However, they do not constrain the problem in practice.

$$
\begin{aligned}
& \text { Find }\left\{\boldsymbol{c}^{(1) *}, \ldots, \boldsymbol{c}^{(L) *}\right\}=\underset{\left\{\boldsymbol{c}^{(1)}, \ldots, \boldsymbol{c}^{(L)}\right\}}{\operatorname{argmax}} \sum_{l=1}^{L} f\left(\boldsymbol{H}_{l}, \boldsymbol{c}^{(l)}\right) \\
& \text { with } 0<c_{l k}^{*}<\sigma_{y_{l k} \mid \mathbf{H}_{l}}^{2} \forall k \in\{1, \ldots, K\}, \forall l \in\{1, \ldots, L\} .
\end{aligned}
$$




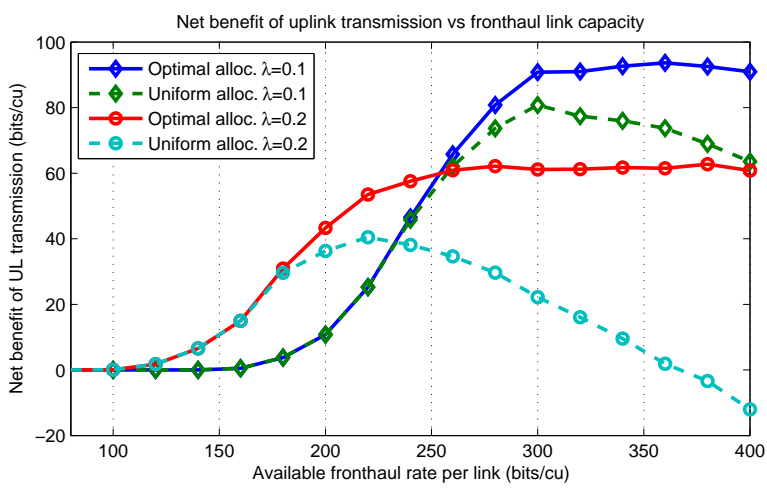

Fig. 2: Net benefit of uplink transmission with constrained fronthaul

\section{Numerical Evaluation of Fronthaul ALLOCATION OPTIMIZATION}

\section{A. System parameters}

We have evaluated the results of the above fronthaul allocation optimization (see equations (17) and (19)) with $N=40$ users located at the edge of 2 cells with 1 RRH each. Note that the optimization problem can be solved efficiently using standard convex programming [9]. We have $K=8$ antennas equally distributed between the RRHs. Channel realizations are modeled using independent one-ring scatterer model for each user [10]. Users are transmitting jointly on the uplink by groups of 4 users each, each user group having its own RB.

\section{B. Efficient transmission with constrained fronthaul}

In a scenario where we aim to find the optimal fronthaul allocation scheme in presence of limited fronthaul links, one can be interested to evaluate if the available amount of fronthaul capacity allows an efficient transmission. Furthermore, while designing fronthaul infrastructures, the evaluation of required capacity to get optimal benefit from the transmission with expected price values $\left(\lambda_{k}\right)$ can contribute to correct the dimensioning of the links to be deployed.

In Figure 2, we compare the proposed fronthaul allocation scheme that optimizes the net benefit of the transmission against uniform fronthaul allocation for different values of available fronthaul capacity. In case of uniform fronthaul allocation, available fronthaul capacity is equally distributed between all groups and all antennas. When available fronthaul is low, both uniform and optimized allocation result in similar efficiency, since the constraint does not allow to achieve higher sum rate. One can notice that optimized fronthaul allocation allows to achieve higher transmission benefit, since in case of sufficient fronthaul, group sum rates can be improved by allocating more fronthaul to the received signals with higher powers. In other words fronthaul allocation is adapted to the variations of channel gains for different users and antennas. Even in an ideal scenario with $\lambda_{k}=0.1$ and a relatively high available fronthaul rate, e.g., 300 bits/channel use, the proposed allocation scheme increases net benefit

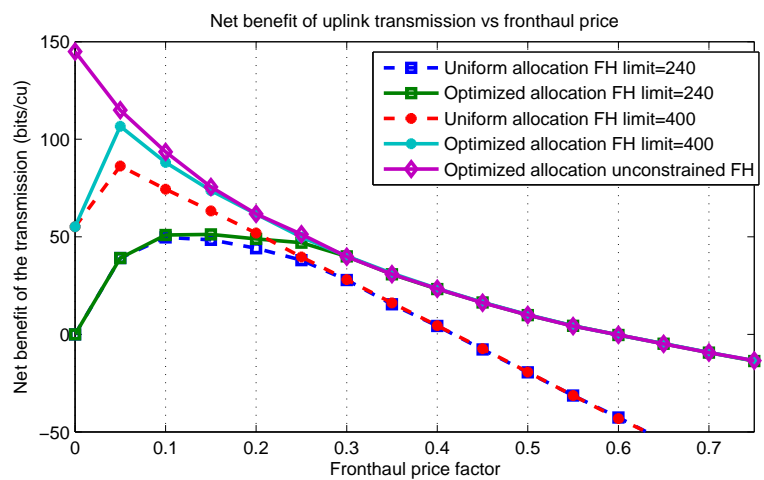

Fig. 3: Net benefit of uplink transmission with various fronthaul constraints vs. fronthaul price factor

of the transmission by approximately $10 \%$. Furthermore, by optimizing the fronthaul allocation, in the operation regime where the sum rate is limited by the transmission power, allocated fronthaul does not continue to increase so that the cost remains moderate. In uniform allocation cost continues to increase while the sum rate is limited, which result in the drop of the benefit that we can observe in Figure 2. Obviously, if the price of the fronthaul usage is higher, the transmission is less efficient for both allocation strategies, since the maximal sum rate does not increase while the cost does.

\section{At what price is it still useful to transmit?}

When fronthaul capacity has a fixed limitation (including practically infinite capacity available), the net benefit of the transmission can turn to negative values when uniform allocation scheme is used and cost is too high. By optimizing capacity allocation, we can avoid having higher fronthaul cost than the utility of the transmission, i.e., the sum rate. However, reducing allocated fronthaul rate when the price factor is high would result in a decrease of the sum rate. Depending on the transmission requirements, setting a lower bound on the sum rate that allows to achieve the required quality-of-service can be used to select whether it is useful to transmit with a given price or not.

We can see in Figure 3 that the more expensive the fronthaul is, the more we improve transmission benefit by fronthaul optimization since we can avoid allocating very high cost fronthaul rate. Consequently, optimized fronthaul allocation can reduce the sum rate of the transmission, as we can observe in Figure 4. If the sum rate is very low because of low FH rate that should be allocated, it can be more reasonable to not to transmit. One can also note that the optimal sum rate and net benefit values are almost equal for different fronthaul constraints. A gap can be observed only for low pricing and low available fronthaul rate (see the curve FH limit $=240$ ), since we cannot achieve high benefit despite the optimal fronthaul allocation. 


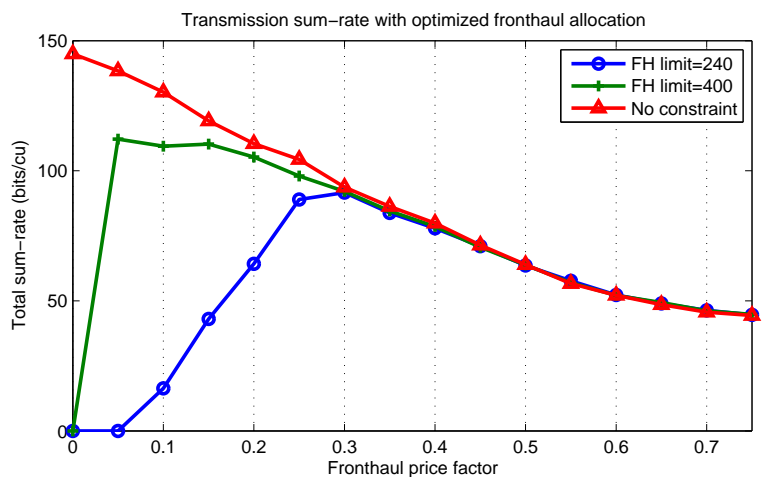

Fig. 4: sum rate of uplink transmission with various fronthaul constraints vs. fronthaul price factor

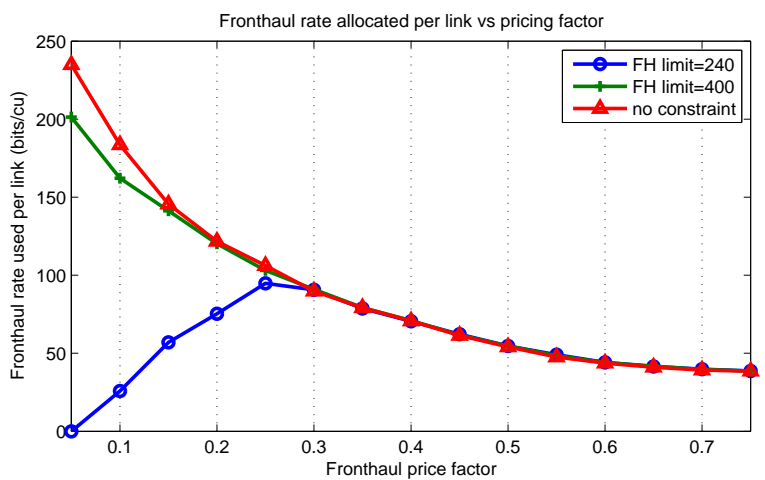

Fig. 5: Fronthaul rate allocated per link against price factor

\section{Impact of the price on FH link usage}

We compare the amounts of allocated fronthaul rate per link with different constraints (including infinite) and varying fronthaul price in order to evaluate the difference between constrained and unconstrained optimization strategies. We can observe in Figure 5 that if available fronthaul rate is high enough (see the curve FH limit $=400$ ) to enable to achieve maximal sum rate, the amount of optimally allocated fronthaul is almost the same with and without the constraint on available fronthaul for any value of the price factor. However, with less fronthaul rate available, obviously less of it is allocated per link even with a low price. For higher price values, we get similar results regardless of the amount of available $\mathrm{FH}$ rate. The reason is that under low fronthaul price, the sum rate term dominates the optimization, so that it is highly motivated to increase the sum rate in the optimization, however it will be limited by the total amount of available fronthaul. For higher price factor, fronthaul cost must be kept low in order to maximize the benefit of the transmission, so the allocated fronthaul will become similar when the price increases with and without constraint.

\section{CONClusion And Future Directions}

A main limitation of C-RAN architecture is the capacity limited and expensive fronthaul links. In this paper, we have analyzed a low complexity multi-user multi-cell transmission scheme where fronthaul rate allocation is performed to maximize uplink throughput. We provide a novel system model where constrained NOMA is used for several cells and multi-antenna RRHs are connected to a CO where UL JD is performed. To optimize the gain with respect to the limited fronthaul capacity and also price, we allocate the $\mathrm{FH}$ rate based on a performance metric exploiting uplink channel gains. We evaluate the net benefit of the transmission which allows a tradeoff between uplink sum rate including practical parameters and the cost of fronthaul usage.

A future work is to explore possible further improvement of the system performance by associating in each group users that can achieve higher sum rate together, i.e., user grouping optimization. This would be challenging due to the increased system complexity and also the fact that only limited channel state information is available before the radio transmission scheduling. However, we can expect further performance gains by adding the optimization of user association.

\section{REFERENCES}

[1] F. Boccardi, R. W. Heath, A. Lozano, T. L. Marzetta, and P. Popovski, "Five disruptive technology directions for 5G," IEEE Communications Magazine, vol. 52, no. 2, pp. 74-80, February 2014.

[2] Nokia, "5G use cases and requirements," 2015.

[3] M. Al-Imari, P. Xiao, M. A. Imran, and R. Tafazolli, "Uplink nonorthogonal multiple access for $5 \mathrm{G}$ wireless networks," in 11th International Symposium on Wireless Communications Systems (ISWCS), Aug 2014, pp. 781-785.

[4] Y. Rui, H. Hu, H. Yi, and H. H. Chen, "Robust user pairing algorithm under channel estimation errors for uplink virtual multiple-input multiple-output systems," IET Communications, vol. 6, no. 3, pp. 318323, February 2012.

[5] L. Liu, S. Bi, and R. Zhang, "Joint power control and fronthaul rate allocation for throughput maximization in OFDMA-Based Cloud Radio Access Network," IEEE Transactions on Communications, vol. 63, no. 11, pp. 4097-4110, Nov 2015.

[6] J.-K. Kang, O. Simeone, J. Kang, and S. Shamai, "Joint signal and channel state information compression for uplink network MIMO systems," in Global Conference on Signal and Information Processing (GlobalSIP). IEEE, 2013, pp. 875-878.

[7] D. Boviz and S. Yang, "Optimal fronthaul capacity allocation and training for joint uplink receiver in C-RAN," in European Wireless, May 2016.

[8] D. Boviz and Y. El Mghazli, "Fronthaul for 5G: low bit-rate design enabling joint transmission and reception," in IEEE Global Telecommunications Conference (Globecom), 5G RAN Design Workshop, December 2016.

[9] S. Boyd and L. Vandenberghe, Convex Optimization. New York, NY, USA: Cambridge University Press, 2004.

[10] D.-S. Shiu, G. J. Foschini, M. J. Gans, and J. M. Kahn, "Fading correlation and its effect on the capacity of multielement antenna systems," IEEE Transactions on communications, vol. 48, no. 3, pp 502-513, 2000 . 